\title{
Design of Detachable Marking Angle Model for Turning Tool
}

\author{
Liu Bo ${ }^{1,}$ and Dai Yuehong1,b
}

${ }^{1}$ Department of mechanical engineering of ZhenJiang College, Zhenjiang, Jiangsu,China

a zjgzliubo@163.com, b105010155@qq.com

Keywords: turning tool, marking angle, model

\begin{abstract}
Turning tool angle is a critical and difficult point during teaching. To help students understand the geometric angle of turning tool, the author designs a detachable marking angle model for the turning tool. The model can be used to assist teaching, which is helpful for visualization. Therefore, the abstract concept can be easily solidified and understanding by students. The teaching effect will be improved accordingly.
\end{abstract}

\section{Objective}

Cutting \& Machining is everywhere in the production of parts, which is actually by way of relative movement between tools and the workpieces. And the tool is the primary element. The effect of machining with tools depends on tool material and the angle of tool. Turning tool angle is a critical and difficult point during teaching. The terms and concepts in this field are huge. They are combined together making it much complicated. What's the worse, angle comprised of point, line and face, plus relative knowledge of mechanical drawing for drawing reading make it harder for student to understand. That's why teachers prescribe it with models. At present, the models used in class are mostly of complicated structure and inconvenient operation, heavy and difficult to be dismantled. So, the author designed a detachable marking angle model for the turning tool for class teaching, which is helpful for visualization. Therefore, the abstract concept can be easily solidified and understanding by students. The teaching effect will be improved accordingly. The cost is relatively low and easily operated. 


\section{Design \& Making}

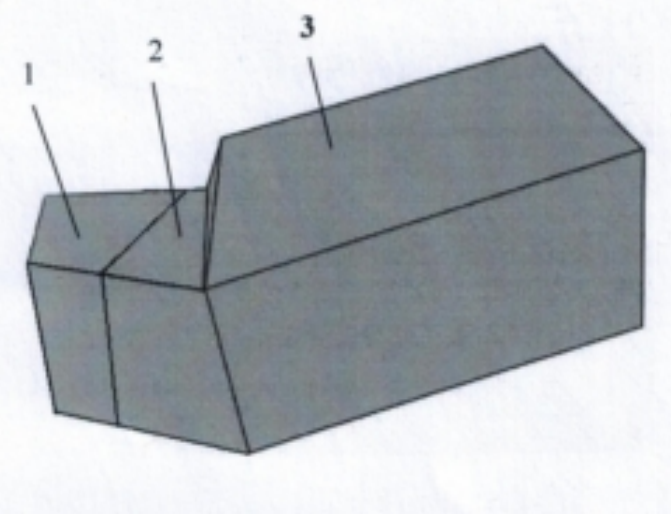

Fig. 1 3D View of Tool 1. blade 2. Blade 3. Handle

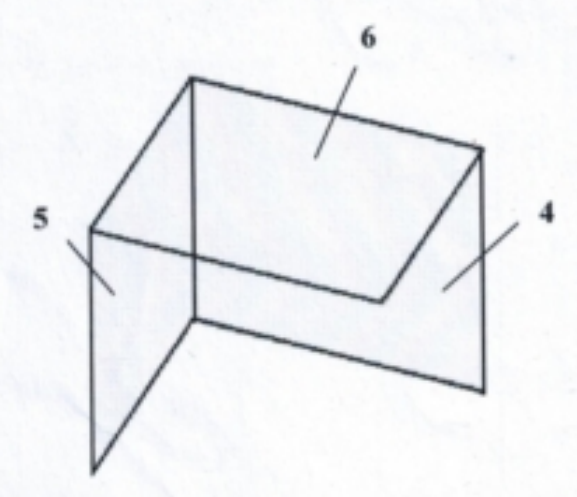

Fig. 2 Stationary reference

4. Base plane 5. Main cutting plane

6. Orthogonal plane

The turning tool is mostly common cutting tool, while other cutting tools can be taken as variants of combination of lathe tool, which is quietly typical. The external tool is the mostly representative tool. Refer to Sketch 1, the external tool is made of wood, form or other materials. Lathe tool is composed of blade and handle. The former has tool 1 and tool 2, which can be joggled or magnetically connected with each other. The contact between tool $1 \& 2$ are shown with section line.

In order to determine the physical position of each tool face and cutting blade, the reference is required. It is used as reference of geometric parameter when tools are designed, produced, grinded and measured, that is stationary reference. Marking angle is the angle used for tool design, grinding and measurement. The stationary reference is composed of three mutually perpendicular assisting faces. Refer to Sketch 2, the three faces are made of organic glass or other transparent materials.

\section{Implementation method}

When in use, show as a whole the tool in Sketch 1 students for them to know the cutting part composed of three faces, two sharp edged and one tip. Then, separate the tool 1 from the tool 2 and install the three auxiliary planes shown in Sketch 2, which coincide with the tool, as shown in Sketch 3. On this basis, explain the establishment of stationary reference and the formation process of tool marking angle.

Angles measured in an orthogonal plane: the front angle $\gamma_{0}$ and rear angle $\alpha_{0}$. Separate the tool 1 from the tool 2, that is, remove the 2 and 3 as shown in Sketch 4. The concepts of the front and rear angle are clear then. 3. The angle between the front and the base surface is the front angle, and the angle between the main rear and the main cutting plane is the rear angle.

From the angle measured in the base plane: the base plane 4 is projection plane of main cutting edge angle $\mathrm{k}_{\mathrm{r}}$ and the auxiliary cutting edge angle $\mathrm{k}_{\mathrm{ro}}$ and by way of rthographic projection principle, 
set front face base plane as projection. The angle of cutting edge angle is defined by the feed direction. The angle between the main cutting edge and the feed direction is the main cutting edge angle. And the angle between the auxiliary cutting edge angle and the feed direction is the auxiliary cutting edge angle

Angle measured in the main cutting plane: cutting edge angle $\lambda \mathrm{s}$. The angle between the main cutting edge angle and the base plane is the cutting edge angle. It is clearly found in the main cutting plane 5 .

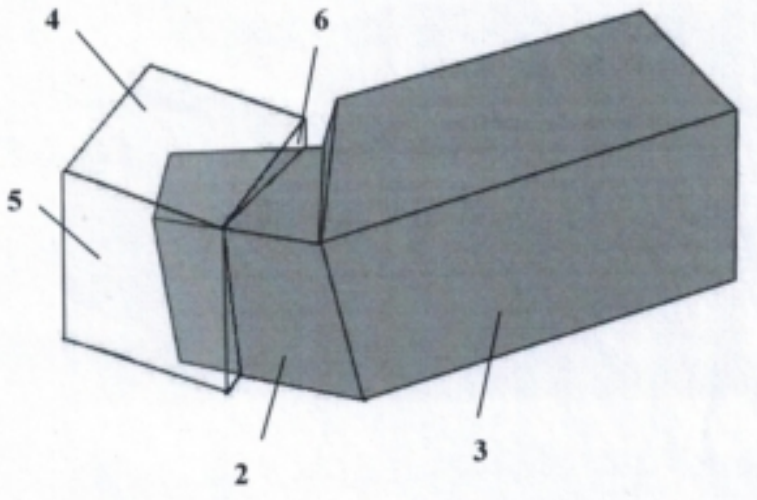

Fig.3 Tool Marking Angle Structure 1. blade 2. Blade 3. Handle 4. Base plane 5. Main cutting plane 6. Orthogonal plane

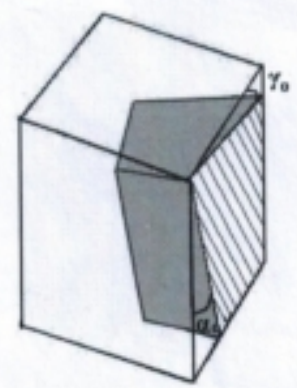

Fig.4 Tool Section

\section{Implementation effect}

The advantage of this model is that it is light and removable. Because all tool angle parameter are defined in the space, the three auxiliary planes are to determine the angle of turning tool and the imaginary plane, which do not exist. They are abstract as well, It is difficult for students to understand. With the assistance of the model, we can specify the abstract concept of space and help students understand the geometric angle of tool, so as to improve the teaching efficiency.

\section{Acknowledgements}

The paper is one of the results of China metallurgical education institute of higher education research key research fund project (2016-2018) (Project Code:2016ZYJHKT-B018) and Project of philosophy and social science research fund of Jiangsu University of 2017(Project Code:2017SJB1118) .

\section{Reference}

[1]Fuchang JIN. Lathe Tool for Beginners, 2 version [M]. Beijing: China Machine Press, 2012 [2]Yanmin PU, Xiaohong LI. Selection \& Sharpneing of Cutting Tool [M]. Beijing: Chemical industry Press, 2012

[3] Binheng LU. Foundation to Machining Technology [M]. Beijing: China Machine Press, 1999

[4]Baojing DI. Computer Aided Test Instruction of "Establishment of Tool Angle Measurement \& Marking Angle" [J]. Modern Manufacturing Technology and Equipment, 2016, 232 (3), 189-190 
[5]Bolong YIN. CAD/CAE Analysis of Geometrical Angle of Digital Tool based on CREO [J].Journal of Bengbu University, 2016, 5 (2), 4-7

[6]Wanxin WANG. Digital Display Study of Tool Angle Measurement [J]. Manufacturing Technology \& Machine Tool, 2015, 2, 83-85

[7]Xiaoliang FAN, Jiaojiao ZHANG. Design of Aided Education System of Geographic Tool Angle [J]. China Mechanical Engineering, 2011, 1，52-54

[8]Chunjiao LV. Cracking "Tool Angle" Education Puzzle with Multiple Methods [J]. Private Technology, 2011, 2, 85 